\title{
Agency and Ownership are Independent Components of 'Sensing the Self' in the Auditory-Verbal Domain
}

\author{
Daniela Hubl • Rahel C. Schneider • \\ Mara Kottlow • Jochen Kindler • Werner Strik • \\ Thomas Dierks $\cdot$ Thomas Koenig
}

Received: 18 September 2013/ Accepted: 11 January 2014/Published online: 28 January 2014

(C) Springer Science+Business Media New York 2014

\begin{abstract}
Sensing the self' relies on the ability to distinguish self-generated from external stimuli. It requires functioning mechanisms to establish feelings of agency and ownership. Agency is defined causally, where the subjects action is followed by an effect. Ownership is defined by the features of the effect, independent from the action. In our study, we manipulated these qualities separately. 13 righthanded healthy individuals performed the experiment while 76-channel EEG was recorded. Stimuli consisted of visually presented words, read aloud by the subject. The experiment consisted of six conditions: (a) subjects saw a word, read it aloud, heard it in their own voice; (b) like a, but the word was heard in an unfamiliar voice; (c) subject heard a word in his/her own voice without speaking; (d) like c, but the word was heard in an unfamiliar voice; (e) like a, but subjects heard the word with a delay; (f) subjects read without hearing. ERPs and difference maps were computed for all conditions. Effects were analysed topographically. The N100 (86-172 ms) displayed significant main effects of agency and ownership. The topographies of the two effects shared little common variance, suggesting independent effects. Later effects $(174-400 \mathrm{~ms})$ of agency and ownership were
\end{abstract}

Electronic supplementary material The online version of this article (doi:10.1007/s10548-014-0351-0) contains supplementary material, which is available to authorized users.

D. Hubl ( $($ ) · R. C. Schneider · M. Kottlow · J. Kindler .

W. Strik - T. Dierks · T. Koenig

Department of Psychiatric Neurophysiology, University Hospital

of Psychiatry, Bolligenstrasse 111, CH-3000 Bern 60,

Switzerland

e-mail: hubl@puk.unibe.ch topographically similar, suggesting common mechanisms. Replicating earlier studies, significant N100 suppression was observed, with a topography resembling the agency effect. 'Sensing the self' appears to recruit from at least two very distinct processes: an agency assessment that represents causality and an ownership assessment that compares stimulus features with memory content.

Keywords Language · Self-monitoring · Corollary discharge $\cdot$ Healthy controls - Auditory evoked potential . N100 · Topography

\section{Introduction}

Credible indexing that 'I am the initiator of my actions' is important for distinguishing self-generated stimuli from external stimuli. In the 1950s, Sperry (1950) and Holst (1954) followed the early ideas of Helmholtz (1924) and Hughlings Jackson (1958) of a "motor theory of thought". They suggested a motor-driven efference copy mechanism towards the sensory brain areas that allows the detection of differences between incoming sensory feedback with the internal representation. The predicted sensory consequence is called corollary discharge (Ford and Mathalon 2012). An important paper, which consolidated these concepts of efference copy and corollary discharge in the psychiatric literature and linked it explicitly with psychotic symptoms was the work of Feinberg (1978), who hypothesized that this mechanisms of control and integration are not only present in the motor system but also in thinking. He speculated that the derangement of corollary discharge could produce many of the symptoms of schizophrenia.

Although the terms efference copy and corollary discharge are often used interchangeably or jointly (Feinberg 
1978), some authors (Crapse and Sommer 2008; Ford and Mathalon 2012) distinguished between them. "Efference copy" is used to refer to a copy of the motor command and "corollary discharge" to the expected sensation resulting from this particular action. Beside the human studies, research in many species suggests that these mechanisms allow all species in the animal kingdom to tag sensations as coming from "self" (Crapse and Sommer 2008).

The idea of sensory predictions based on an efference copy is discussed for sensory-motor integration in modalities such as visual (Wurtz 2008), auditory-verbal (HeinksMaldonado et al. 2007; Houde and Jordan 1998), and somatosensory systems (Blakemore et al. 2002). It also is believed that the brain generally uses the same mechanisms for visuo- (Leube et al. 2003) or audio-motor (Maidhof et al. 2010) integration. These predictive mechanisms help us to distinguish our own actions from those of others.

An excellent example of a deficient system of sensing the self is observed in individuals with psychotic disorders such as schizophrenia. In hearing voices, thought insertion, withdrawal, made volition, impulses or affects, derealisation and depersonalisation, a lack of the ability to correctly distinguish self-generated from external stimuli, might be part of the neurophysiological deficit. Certainly, other specific deficits also contribute to the respective full symptom (e.g. a pathological activation of the auditory cortex in auditory verbal hallucinations) (Dierks et al. 1999).

In schizophrenia, core symptoms are found in the verbal domain. Therefore, our main interest is in predictive mechanisms in the verbal system. In healthy subjects, the intact functions of predictive verbal mechanisms are important for the acquisition of speech and speech fluency. Neurobiologically, these mechanisms are realized by an intact and cooperating motor and sensory language system. Specifically, an efference copy from the frontal motor speech area is sent towards the specific temporo-parietal sensory areas. The efference copy of the motor speech command predicts the sensory consequences, namely the corollary discharge, and consequently, what will be heard. Subsequently, the brain compares the corollary discharge with the actual incoming acoustic stimulus. In case of consistency, the internal prediction matches the self-generated sensory input. As a consequence, the activation of the auditory cortex is dampened, and in healthy controls the suppression of the electrophysiological N1 component (i.e. the answer of the primary auditory cortex to an acoustic stimulus) reflects the consequences of the corollary discharge generated during speaking and listening by the efference copy (Houde et al. 2002; Pantev et al. 1988).

It has been demonstrated that the N1 responses to a selfproduced and, thus, expected vowel/a/were weaker than responses to the same, tape-recorded/a/. This finding supports the hypothesis of an exact prediction of the expected incoming stimulus by the efference copy (Houde et al. 2002). When feedback has been manipulated by giving back a stimulus that does not exactly match the expectation (e.g. altering or replacing the voice speaking/a/), the N1 suppression is reduced or even missing (Heinks-Maldonado et al. 2005). This suppression seems to depend on the degree of the alteration of the stimulus: Matching and thus expected stimuli lead — as we already know — to a maximal suppression. Alien or maximal altered stimuli go along without suppression, and stepwise graded altered stimuli merge with the amount of alteration into a stepwise diminishing suppression (Behroozmand and Larson 2011).

Intact efference copying implies that the initiator of an act owns the results of it. To understand the processes contributing to this complicated system, we introduce definitions of two comprised concepts, namely agency and ownership. The proposed definition of agency is causal; the subjects action (being the cause) is followed by an effect within a precise time window. This does not imply much about the nature of the effect: For example, if I push the light switch, and the TV, but not the light goes on, I still feel I have caused it, albeit the effect was unexpected. Ownership is defined by the features of the effect, independent from the action of the subject. I may for example immediately recognize my writing, even if I'm not able to remember that I wrote it. In a healthy condition, the subject attributes both agency and ownership to the effects of its own actions: There is a strong feeling of having initiated the effect, and the effect contains features that unequivocally attribute it to past, self-initiated experiences of a similar kind. In the context of our experiment, agency was therefore defined as hearing a word immediately after reading a word, independent of the acoustic features of the heard word. On the other hand, ownership was defined as whether the stimulus contained acoustic features that clearly attributed it to the past experiences of hearing one's own voice. Agency was thus assumed to be present if and only if the subject's auditory experience was precisely time-locked to the subject's act of speaking, and absent if such a precise temporal relation was absent or distorted (here: delayed). Ownership was assumed to be present if and only if the subject heard a word with her/his own voice, and absent when hearing an unfamiliar voice.

Our distinctive definitions of agency and ownership are interesting also in the context of a recently proposed theoretical framework (Synofzik et al. 2013) that distinguishes, when explaining "feeling of self", predictive processes that are based on causality and assumingly yield a "feeling of agency", and post-dictive processes, that compare sensory input with memory based information. These post-dictive processes were assumed to correspond to a "judgement of agency", which contains our definition of "ownership". 
In summary, behaviourally evident functional and dysfunctional self-monitoring seems to depend on several biological mechanisms (Johns and Mcguire 1999). These mechanisms may independently yield a sense of ownership and agency of perceptions. In the present study, we introduce a novel experimental design to disentangle the biological substrates of agency and ownership contributing to the 'sense of self'. We aimed to investigate the contribution of these two processes on 'sensing the self' by analysing event related potential (ERP) components from a healthy control sample. We hypothesized that the two processes are different from each other as seen by different topographies and that both processes contribute to an intact recognition of self-caused actions.

\section{Materials and Methods}

\section{Subjects}

Subjects included 13 healthy right-handed adults (6 men), ranging in age from 19 to 34 years (mean $=24.1$, $\mathrm{SD}=3.4$ ). Medical and psychiatric disorders were assessed by questionnaire and served as exclusion criteria. No subjects reported a history of auditory, visual, psychiatric, or neurological disorders. All study procedures, including recruitment, data acquisition, and informed consent, were arranged by the Department of Psychiatric Neurophysiology of the University Hospital of Psychiatry Bern. Subjects were compensated with a voucher for their participation. Before the electrophysiological measurements, subjects passed the Whispered-Voice Test (Macphee et al. 1988) to screen for hearing impairments. All subjects provided written informed consent and the study was conducted in accordance with the Declaration of Helsinki. Study protocol was approved by the local ethics committee of the Canton of Bern.

\section{Experimental Design and Stimulus Material}

The experiment allowed separating the effects of agency and ownership on auditory word perception and eliminate the effects of overt speech production. In its basic form, it consisted of visually presented words that had to be read immediately, and instantly perceived by the reader/speaker. However, due to reasons described below, not all stimulus conditions contained both a visual and an auditory component (Table 1).

The experiment consisted of the following 6 conditions: (a) subjects saw a word, read it aloud, and immediately (without delay) heard it in their own voice (Normal); (b) subjects saw a word, read it aloud, and immediately heard it spoken by another, unfamiliar voice (Unfamiliar);
Table 1 The stimulation conditions: The six conditions are listed and briefly described

\begin{tabular}{|c|c|c|c|}
\hline Name & Description & Agency & Ownership \\
\hline Normal & $\begin{array}{l}\text { Subjects saw a word, read it } \\
\text { aloud, and immediately heard } \\
\text { it in their own voice }\end{array}$ & + & + \\
\hline Unfamiliar & $\begin{array}{l}\text { Subjects saw a word, read it } \\
\text { aloud, and immediately heard } \\
\text { it, but spoken with an } \\
\text { unfamiliar voice }\end{array}$ & + & - \\
\hline $\begin{array}{l}\text { Feedback- } \\
\text { Only }\end{array}$ & $\begin{array}{l}\text { Without seeing and reading a } \\
\text { word, the subject heard him/ } \\
\text { herself speaking a word }\end{array}$ & - & + \\
\hline $\begin{array}{l}\text { Unfamiliar- } \\
\text { Only }\end{array}$ & $\begin{array}{l}\text { Without seeing and reading a } \\
\text { word, subjects heard an } \\
\text { unfamiliar voice speaking a } \\
\text { word }\end{array}$ & - & - \\
\hline Delay & $\begin{array}{l}\text { Subjects saw a word, read it } \\
\text { aloud, and heard the word in } \\
\text { their own voice, but with a } \\
\text { delay of } 200 \mathrm{~ms}\end{array}$ & \pm & + \\
\hline $\begin{array}{l}\text { Read- } \\
\text { Aloud- } \\
\text { Only }\end{array}$ & $\begin{array}{l}\text { Subjects saw a word and read it } \\
\text { aloud, but received no } \\
\text { auditory feedback. This } \\
\text { condition was used to control } \\
\text { for speech-related artefact }\end{array}$ & - & - \\
\hline
\end{tabular}

Agency and ownership effects are indicated for each condition with + for the presence of the respective effect and - for the absence. In the Delay condition the presence of a distorted the agency effect is indicated with \pm

(c) without seeing and reading a word, the subject heard him/herself speaking a word (Feedback-Only); (d) without seeing and reading a word, subjects heard an unfamiliar voice speaking a word (Feedback-Unfamiliar); (e) subjects saw a word, read it aloud, and heard the word in their own voice, but with a delay of $200 \mathrm{~ms}$ (Delay); and finally, (f) subjects saw a word and read it aloud, but received no auditory feedback (Read-Aloud-Only). This condition was used to control for speech-related artefact elimination (Table 1).During the experiment, the subjects wore headphones and sat in a comfortable resting position in front of computer monitor (distance $70 \mathrm{~cm}$ ) and a microphone in a slightly darkened room. Throughout the experiment, a fixation cross was shown in the centre of the screen except for when a word was presented. Visual stimuli consisted of 270 two-syllable, concrete, and neutral nouns. We controlled for equality in frequency, word length, and gender (male, female, neutral) within and among the blocks. The words and their concreteness ratings were selected from a word list from Wirth (Wirth et al. 2008, 2011). In each trial that included a visual word presentation, the word was shown for one second (visual angle of a 5-letter word was 4.9 degrees). There were a total of 90 trials in each of the six conditions, making a total of 540 trials. Each of the 270 
words was therefore employed twice in the same condition, except of the words from conditions Normal and FeedbackOnly where 90 different words appeared in each of the two conditions, but where identical for both conditions. In the Unfamiliar condition, the subject's voice onset was detected through the microphone, which triggered the playback of the visually presented word read by an unfamiliar speaker. There was no intentional delay between voice onset and the playback of the unfamiliar voice and subjects reported hearing the feedback in "real-time" without perceptible delay. The auditory stimuli for the Feedback-Only condition consisted of the words read (and recorded) by the subject during the Normal condition. The auditory stimuli for the Feedback-Unfamiliar condition consisted of words spoken by a local speaker who was the same sex as the subject (recorded before the experiment).

Each trial consisted of a 1-s pre-stimulus period with only the fixation cross, followed by the presentation of a visual stimulus and an auditory feedback, or by the presentation of an auditory stimulus. Subjects were instructed to read each word on the screen aloud into the microphone. Trials were presented in a pseudo-random order over the duration of the whole experiment and separated by $3 \mathrm{~s} ; 10$ short breaks were given during the experiment. The entire experiment lasted about $35 \mathrm{~min}$. For the analysis of the behavioral data, the delays between stimulus and voice onset time were individually collected in the Normal and Unfamiliar conditions and averaged first within, then across subjects.

To mask unwanted direct auditory feedback, we used a Sennheiser HME 110 headphone (designed for helicopter pilots) to effectively dampen external auditory input. To avoid feedback through bone conduction, a constant pink (1/f) background noise was constantly played through the headphones. Prior to the experiment, noise volume was individually adjusted to be loud enough to mask bone conductions when subjects spoke normally wearing the headphones. Under experimental conditions, the loudness of the presented word was set to $20 \mathrm{~dB}$ above the individual pink noise level.

\section{Electrophysiological Recordings}

The electroencephalogram (EEG) was recorded using silver/silver chloride electrodes attached to the scalp at 74 regularly spaced standard positions of the international extended ten-twenty system. Impedances were kept below $20 \mathrm{k} \Omega$. For artefact monitoring, two additional EOG electrodes were placed below each eye. F3 and F4, electrically separated by buffer amplifiers, served as recording references. Before starting the experiment, a 4-min resting state EEG was recorded for clinical evaluation. During the entire experiment, the EEG was continuously digitized (bandpass filter $0.016-150 \mathrm{~Hz}, 500 \mathrm{~Hz}$ sampling rate) and stored for offline analysis using a Nihon Kohden Neurofax EEG $1100 \mathrm{G}$ system. The onset of the visual presentation of each stimulus, as well as the voice onset, was marked in the EEG.

\section{Data Pre-Processing}

All EEG data were submitted to an ICA-based correction of eye-movements (Jung et al. 2000). Thereafter, the EEG was recomputed to average reference, and periods with visually detectable remaining artefacts (namely muscle activity and electrode artefacts) were eliminated by an EEG expert. Channels containing excessive artefacts were interpolated using spherical splines. All signals were bandpass filtered between 5 and $18 \mathrm{~Hz}$. Based on the markers given by the stimulation programme, the continuous EEG recordings were segmented into 1,200-ms epochs, beginning $200 \mathrm{~ms}$ before voice/sound onset to 1,000 ms postonset. EEG segments were averaged within each stimulus condition and subject (mean number of included trials per condition was 83, range 64-90). To correct for speechrelated artefacts, the individual mean ERP of the ReadAloud-Only stimulus condition was subtracted from the individual ERPs of the Normal, Unfamiliar, and Delay conditions.

\section{Statistical Analysis}

The grand mean ERP was computed across all conditions (Feedback-Only, Unfamiliar-Only, Normal, Unfamiliar, Delay) and subjects and divided into temporal components based on the grand mean's dissimilarity. The ERP maps were then averaged subject- and condition-wise across time within each temporal component. The peaks of this curve indicated end respectively start point of a particular time window corresponding to separable, stable, and mathematically defined ERP components (Michel et al. 2009).

The statistical comparison of the ERP component maps was based on a randomization procedure called TANOVA (Strik et al. 1998), as implemented in the Ragu programme (Koenig et al. 2011). In brief, a TANOVA uses randomization statistics to compare multichannel ERP. Since a TANOVA considers the entire scalp field as a single entity, significant TANOVA effects imply at least partially different sources of the analysed ERP component maps. The main analysis contrasted the four conditions Normal, Feedback-Only, Unfamiliar, and Unfamiliar-Only along the two orthogonal factors agency and ownership (see Table 1). The factors followed a $2 \times 2$ design with conditions Normal (agency +/ownership +), Feedback-Only (agency-/ownership +), Unfamiliar (agency +/ownership-) and Unfamiliar-Only (agency-/ownership-). One additional one-way TANOVA contrasted the Feedback-Only 
condition against the Normal condition, aiming to replicate the previously reported effects of the feed-forward prediction mechanism (Ford et al. 2001a, b; Heinks-Maldonado et al. 2005).

Finally, the Delay condition was contrasted with the Normal condition to assess its effect.

Where there were significant $(p<0.05)$ TANOVA effects, they were followed up by t-maps to display the spatial distribution of the encountered scalp map difference.

In order to estimate the putative sources of scalp ERP effects attributable to agency or ownership, voxelwise inverse statistics were computed for those components and contrast where the TANOVA was significant. Intracerebral current density was estimated using the sLORETA software (Pascual-Marqui 2002) that used a boundary elements head model derived from the MNI152 template, and a total of 6,239 voxels of $5 \times 5 \times 5 \mathrm{~mm}$ size that covered all cortical gray matter. The regularization parameter was set to a SNR of 100. Paired t-statistics across subjects were used to assess significance of local differences. Since the overall null-hypothesis had had already been rejected before computing a contrast in the inverse space, no further corrections for multiple testing were applied. The obtained $\mathrm{t}$-values were thresholded at $\mathrm{t}$-values corresponding to a two-tailed significance level of $5 \%$. Local maxima and their positions were reported in MNI coordinates.

\section{Results}

\section{Behavioral Data}

Mean voice onset time was $607.9 \mathrm{~ms}$ (sd: $46.1 \mathrm{~ms}$ ) after the onset of stimulus words. This is in close agreement with previous literature (Gould et al. 2012) and indicates that our subjects performed the task as instructed.

\section{Segmentation of the Components}

The temporal segmentation of the EEG components based on spatial similarity as assessed by the correlation coefficient of the grand mean of the five conditions (Fig. 1). This resulted in times periods from 20 to $84 \mathrm{~ms}$ for the early component, $86-172 \mathrm{~ms}$ for the N100, and $174-400 \mathrm{~ms}$ for the late component.

\section{Early Component}

The TANOVA indicated main effects of agency $\left(p=0.040, \mathrm{t}_{\max }\right.$ at $\mathrm{Oz}=3.211, \mathrm{t}_{\min }$ at $\left.\mathrm{FC} 2=-3.381\right)$ and ownership $\left(p=0.006, \mathrm{t}_{\max }\right.$ at $\mathrm{AF} 7=4.715, \mathrm{t}_{\min }$ at $\mathrm{P} 2=-3.203)$ as well as an interaction $\left(p=0.04, \mathrm{t}_{\max }\right.$ at $\mathrm{F} 2=5.020, \mathrm{t}_{\min }$ at $\left.\mathrm{P} 9=-3.699\right)$.

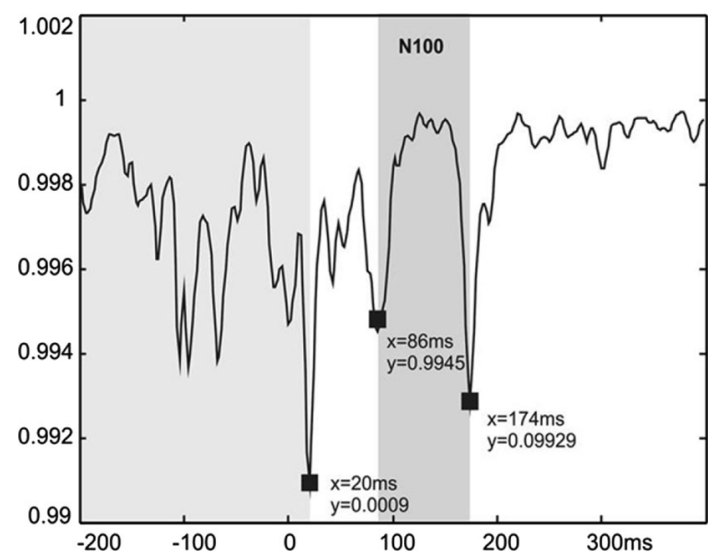

Fig. 1 Temporal segmentation of the ERP components

\section{N100 Effects}

For all conditions, the N100 topography in general resembled the expected configuration with a central negativity and a bilateral temporal positivity (Fig. 2). There were, however, considerable differences between conditions, which were confirmed by significant effects in the two-factorial TANOVA. In particular, the TANOVA indicated that there was a significant main effect of agency ( $p=0.001)$ and ownership $(p=0.033)$, but no interaction between the two factors $(p=0.615)$.

The t-map of the N100 ownership effect (defined by the contrast of the average of Normal and Feedback-Only against the average of Unfamiliar and Unfamiliar-Only; Fig. 3a, middle row) had a bilateral centro-temporal negativity $\left(\mathrm{t}_{\min }\right.$ at $\left.\mathrm{C} 5=-3.549\right)$ and a surrounding positivity $\left(t_{\max }\right.$ at $\left.\mathrm{F} 9=4.283\right)$. The $\mathrm{t}$-map of the N100 agency effect (as represented by the contrast of the average of Normal and Unfamiliar against the average of Feedback-Only and Unfamiliar-Only; Fig. 3b, middle row) had a centro-parietal positivity $\left(\mathrm{t}_{\max }\right.$ at $\left.\mathrm{P} 4=4.187\right)$ and a bilateral frontotemporal negativity $\left(\mathrm{t}_{\min }\right.$ at $\mathrm{FC6}=-3.228$ ). (For the wave forms, see Figure s1 and s2 in the supplementary material).

The topography of the agency effect was clearly different from the topography of the ownership effect $(\mathrm{r}=$ -0.311 , common variance $=9.6 \%$ ). Computing multidimensional scaling, the effects of agency and ownership seemed to be independent and quite orthogonal (Figure s3, supplement).

\section{The Late Component}

Overall, the late component was generally characterized by an occipito-parietal negativity and a bilateral frontal positivity (Fig. 2). Again, the TANOVA indicated significant main effects of agency $(p=0.001)$ and ownership $(p=0.037)$ but no significant interaction $(p=0.667)$. 


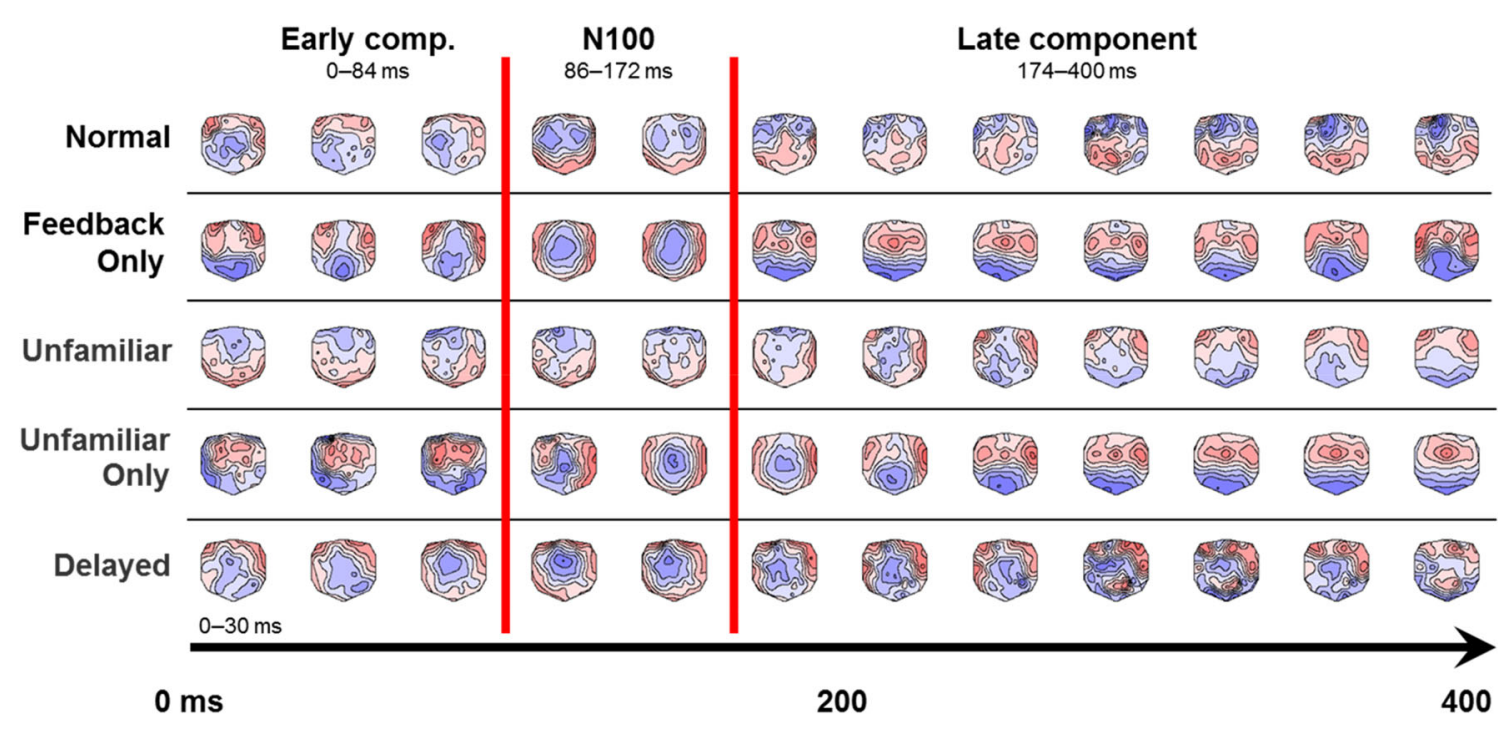

Fig. 2 Mean ERPs topography maps for all five but the control condition. Each color level corresponds to a step of $1 \mu \mathrm{V}$ (Color figure online)

Fig. 3 Main effects of ownership (a) and agency

(b) for the early, N100, and late components

\section{(a) Ownership}

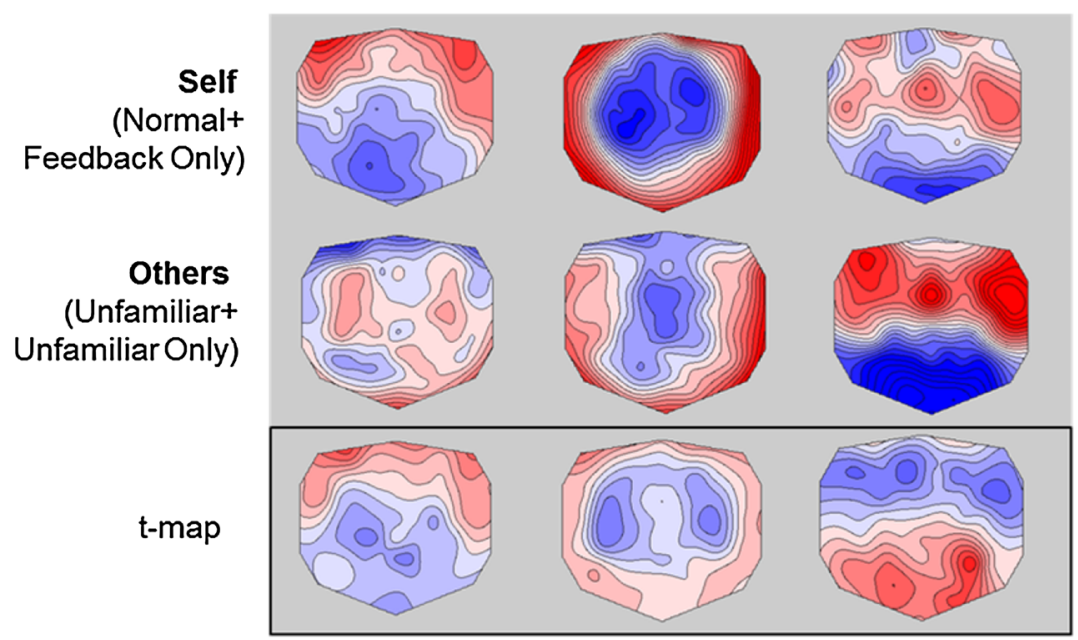

(b) Agency

Expected
(Normal+
Unfamiliar)

Unexpected
(Feedback Only+
Unfamiliar Only)

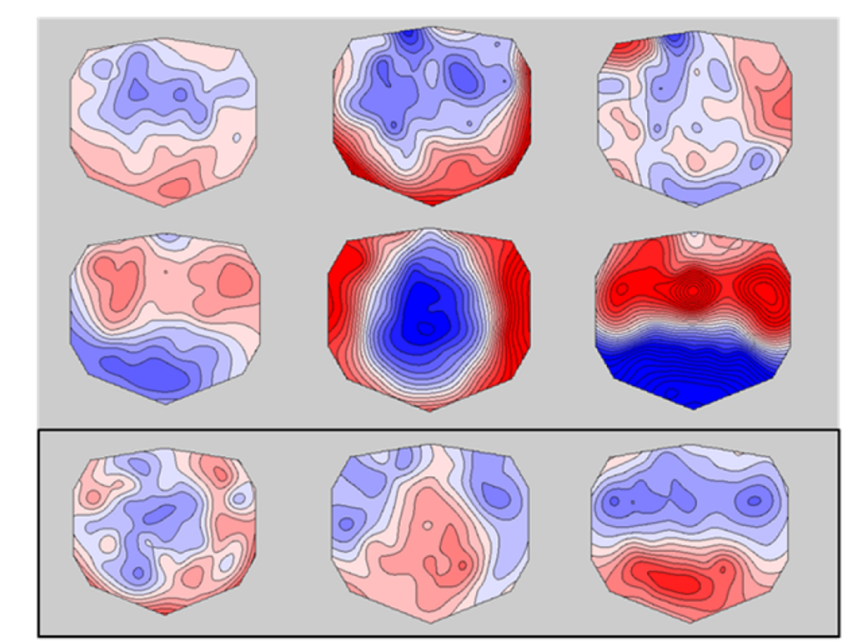

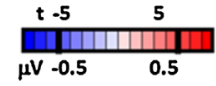

t-map

\section{Early component $\quad \mathrm{N} 100 \quad$ Late component}


(a) Confirmatory Analysis

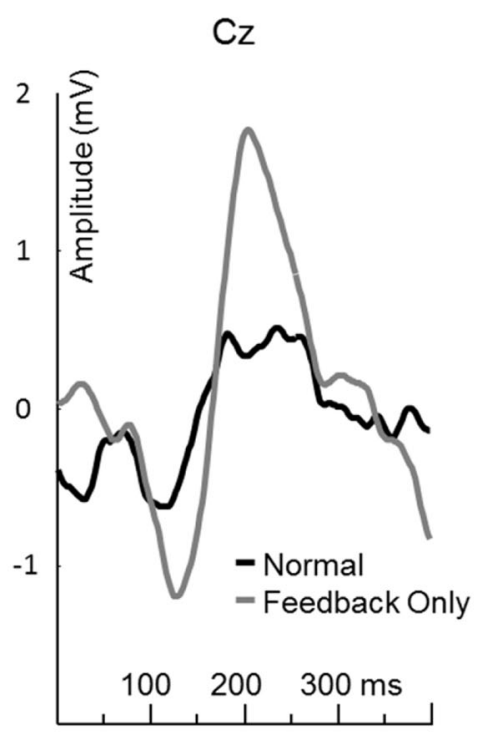

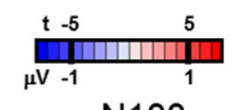

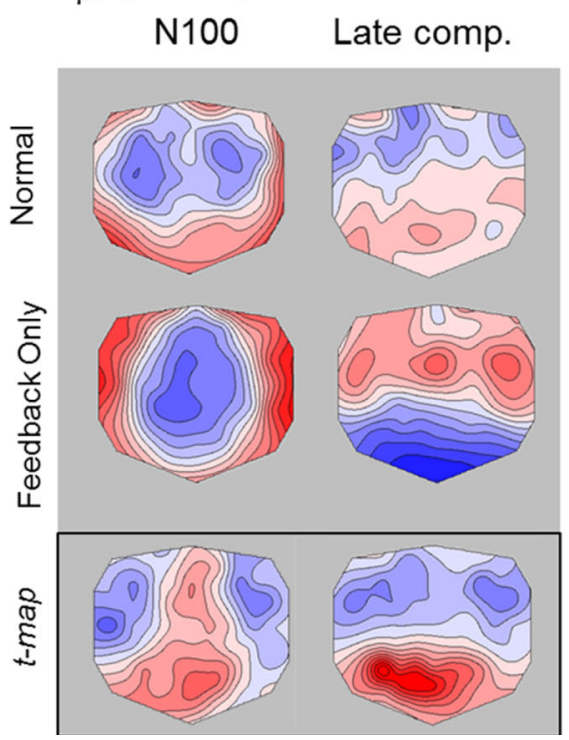

(b) Delay Effect

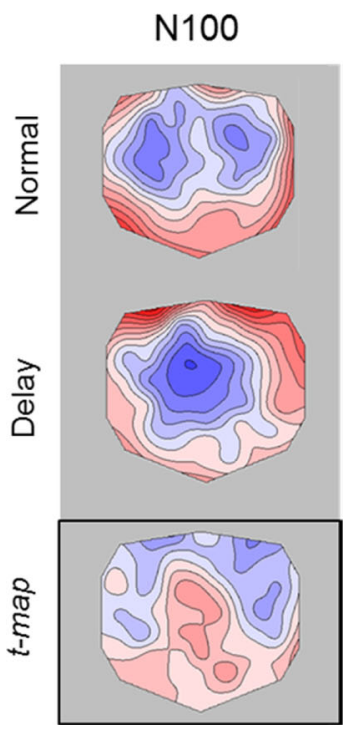

Fig. 4 Topographies of the confirmatory analysis of the N100 suppression (a) as the contrast of the Normal against the Feedback-Only conditions and, second, of the Delay condition (b) where the contrast between the Delay and Normal conditions is shown

The t-map of the effect of agency had a bilateral frontotemporal negativity $\left(\mathrm{t}_{\min }\right.$ at FT7 $\left.=-4.180\right)$ and a parietooccipital positivity $\left(\mathrm{t}_{\max }\right.$ at $\left.\mathrm{POz}=6.877\right)$. The $\mathrm{t}$-map of the ownership effect $\left(\mathrm{t}_{\min }\right.$ at $\mathrm{F} 1=-4.724, \mathrm{t}-\mathrm{max}$ at $\left.\mathrm{P} 6=6.566\right)$ was very similar to the t-map of the agency effect $(r=0.838$, common variance $=70 \%$ ); effects of ownership and agency seemed to be additive (Figure s1, supplement).

\section{Confirmatory Analysis}

To confirm previously published findings, we computed the contrast of the Normal condition against the Feedback-Only condition. This yielded significant TANOVA effects in the $\mathrm{N} 100(p=0.003)$ and the late component $(p=0.001)$, but not in the early component. In the N100 period, the t-map (Fig. 4) closely resembled the t-map of the N100 main effect of agency (Fig. 3b) $(r=0.94$, common variance $=88 \%$; against a common variance of $2 \%$ with the ownership effect) (Fig. 4). In addition the result closely replicated previously reported effects at $\mathrm{Cz}$ in similar experiments (Ford et al. 2001a, b), with a reduction of the N100 amplitude in the Normal condition. In the late component, there also was a significant effect $(p=0.001)$ that resembled the main effects of agency and ownership of the above analyses.

\section{Delay Effect}

The delay effect, defined as the contrast of the Delay condition against the Normal condition showed a significant TANOVA result only in the N100 (but only after normalization of Global Field Power; $p=0.034$ ). As in the confirmatory analysis, the t-map of the delay effect clearly attributed the map difference to agency (common variance $=63 \%$ ) and not to ownership $($ common variance $=2 \%$ ).

Inverse Solutions

sLORETA findings were computed for the main effects of agency and ownership in the N100 component. The results, as shown in Fig. 5, indicated that the presence of agency lead to a decrease of current density estimates in the anterior cingulate cortex including the medial prefrontal cortex $\left(\mathrm{t}_{\max }=4.26, \mathrm{x}=7, \mathrm{y}=11, \mathrm{z}=30\right)$, in the right temporal cortex including the primary auditory cortex $\left(\mathrm{t}_{\max }=3.18, \mathrm{x}=39, \mathrm{y}=-29, \mathrm{z}=19\right)$, and increased current density estimates in the anterior part of the left temporal cortex $\left(\mathrm{t}_{\max }=3.51, \mathrm{x}=-50, \mathrm{y}=5, \mathrm{z}=-\right.$ 15). Presence of ownership increased current density estimates in left and right insula, left inferior frontal gyrus and the anterior parts of the right superior temporal gyrus $\left(\mathrm{t}_{\max }=3.08, \mathrm{x}=50, \mathrm{y}=15, \mathrm{z}=-25\right)$, decreased current density estimates were observed in posterior and rostral anterior cingulate and the occipital cortex $\left(\mathrm{t}_{\max }=3.28\right.$, $\mathrm{x}=5, \mathrm{y}=-90, \mathrm{z}=25$ ).

\section{Discussion}

The distinction of self-generated stimuli from external stimuli is important for an intact sense of self and is 


\section{N100 agency effect}

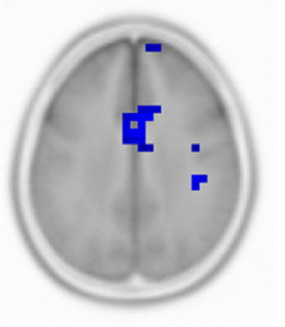

$z=34$

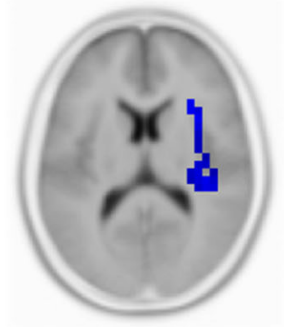

$z=15$

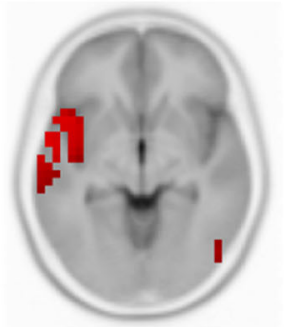

$z=-5$

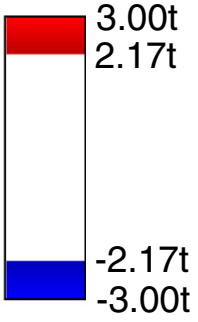

N100 ownership effect

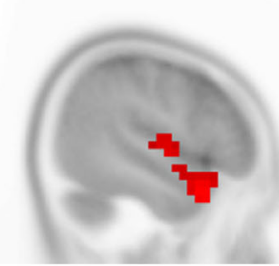

$x=50$

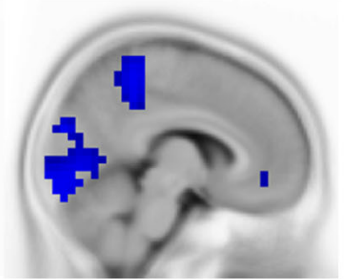

$x=-5$

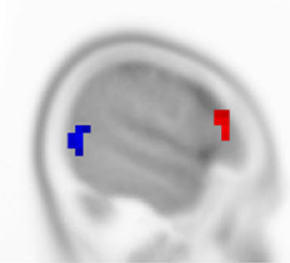

$x=-56$

Fig. 5 Significant N100 effects of agency and ownership in the inverse space. Red areas indicate larger current density estimates under the presence of agency/ownership, blue areas indicate more current density in the absence of agency/ownership (Color figure online)

frequently affected in psychiatric conditions such as psychosis. The present study examined possibly different components of that complex mechanism by defining the processes of agency and ownership from a behavioural/ descriptive viewpoint.

The analysis of the brain electric topography supports that the assumed two components have two consistent and separate biological fingerprints in the N100 period: Consistent, as both components yielded a significant main effect; and separate, as the common topographic variance between the agency effect and the ownership effect was only $9.6 \%$. Thus, 'sensing the self' appears to result from at least two distinct processes: an agency assessment that represents causality and an ownership assessment that compares stimulus features with memory content. This composition of the 'sense of the self' also has been described in other sensory-motor systems. In a recent study using a rubber hand, feelings of control over bodily actions (sense of agency) and the ownership of body parts (sense of ownership) could be behaviourally distinguished, and possibly different underlying neuronal substrates were discussed, paralleling our data which were gained in the auditory and language system (Kalckert and Ehrsson 2012). The distinction between causally defined, predictive processes and processes comparing sensory input with memory content as different elements for a feeling of "selfmade" has also been highlighted in a recent hypothesis and theory article by (Synofzik et al. 2013) who argue that only a context-weighted integration of both yield to a reliable feeling of being the origin of one's actions. Our data suggest that previous papers on central auditory N100 suppression (Ford et al. 2001a, b; Heinks-Maldonado et al. 2005) most likely represented the agency effect. This is supported by our confirmatory analysis that replicated the previously reported central N100 suppression and showed that the topography of this effect was nearly identical ( $88 \%$ common variance) to the main effect of agency, but not to the effect of ownership ( $2 \%$ common variance). Thus, our data confirmed insights from an earlier study trying to 'dissect corollary discharge' in patients with schizophrenia (Ford et al. 2007), where the greatest influence was found in the classical setting of evoking N100 during speaking. No suppression was found when subjects heard their tape-recorded voice self-initiated by a button press or after a visual warning. However, others have found N100 suppression after manually self-initiated sound stimuli and have argued for the existence of monitoring mechanisms other than the innate efference copies (Baess et al. 2011).

Translated to a psychological level, our data indicate that when it comes to the processing of entire words, the central N100 suppression primarily seems to represent the experience of causing an external percept. In other words, if you say a word aloud and you hear the word, the central N100 is suppressed regardless of who is speaking.

The importance of the agency effect for the 'sense of self' is further supported by the results of the Delay condition. Here, the causal relation between action and effect was altered, which again yielded an effect in the N100 topography that matched the agency effect. 
Classically a reduction of the N100 during reading has been attributed to corollary discharge resulting from efference copies of motor commands. This is a common mechanism in humans and animals (Crapse and Sommer 2008). In healthy controls, the largest suppression, and thus smallest N100 amplitudes, are reported for conditions identical to our Normal condition (subject speaks and consequently hears a sound in his/her voice) (Curio et al. 2000; Houde et al. 2002). Responses to self-produced speech were weaker than responses to tape-recorded speech. Further, responses to tones also were weaker during speech production when compared to responses to tones recorded in the presence of speech from tape playback. These data suggest that during speech production, the auditory cortex attenuates its sensitivity and modulates its activity as a function of the expected acoustic feedback (Houde et al. 2002). In studies with patients with schizophrenia, the expected suppression in a normal speaking condition was missing or strongly reduced (Ford et al. 2001ab; Heinks-Maldonado et al. 2007).

The later ERP component seemed to integrate the sense of agency and ownership. The observation that the t-maps of the effects of agency and ownership were very similar (70\% common variance) suggests that both factors contribute to the component in an additive way. Maybe this mirrors a more general mechanism to process unexpected events. In the literature on corollary discharge, to our knowledge, only the N100 effects were described without statistical consideration of the later effects.

In contrast to earlier studies in the field of ERP measurement of auditory self-monitoring, we used entire words (nouns) instead of syllables (Heinks-Maldonado et al. 2005, 2007). This decision was led by our intention to investigate, in subsequent experiments, the mechanisms of identifying stimuli as coming from the self or not in patients with schizophrenia prone to auditory verbal hallucinations. Classically, auditory hallucinations comprise full words, often with semantic or emotional content (Mccarthy-Jones et al. 2012). Therefore, in the behavioural studies of self-monitoring without ERP measurements, emotional words (e.g. adjectives) have been used (Johns and Mcguire 1999). The argument for using emotional words is the idea that auditory verbal hallucinations often include emotional content (Mccarthy-Jones et al. 2012). As we aimed to segregate the different electrophysiological processes regarding agency and ownership, we removed the emotional valence to reduce potential confounding variables. An undesired consequence of using words instead of syllables in electrophysiological studies-which may have prevented others from using this methodologyis movement artefacts. We controlled for movement artefacts by subtracting the Read-Aloud-Only (i.e. speech without listening) control condition from these conditions with the potential speaking artefact (Normal, Unfamiliar, Delay). The topography of our word-evoked N100 was according to N100-literature and had a central negativity, resembling the N100 topography of a syllable (Eichele et al. 2005) or the most commonly used beep tone stimuli (Hubl et al. 2007). Finally, the word evoked N100 replicated the well-described N100 suppression when agency was present (Fig. 4a, left side).

A byproduct of removing speaking artefacts by subtracting the Read-Aloud-Only condition was that feed-forward mechanisms also were removed. Thus, all analysed ERPs represented only auditory processing. In former studies (Heinks-Maldonado et al. 2005, 2007), in the respective speaking condition, the feed-forward mechanisms still were included.

Further, expanding the present literature in the field, we statistically analysed the topographical effects. In the confirmatory analysis of the central N100 suppression (Fig. 4a) the topography of the suppressed N100 had bilateral temporal minima with a slightly stronger pronunciation on the left, speech-dominant hemisphere, corresponding to the figures as presented in an earlier study (Heinks-Maldonado et al. 2007). This likely is due to the corollary discharge invoked by the efference copy in the speaking condition (Normal) following the articulation of a word, which is missing in the listening condition (Feedback-Only). Since the unsuppressed N100 evoked by the listening condition had the typical central negativity, the difference map had strongest amplitudes along the midline. In contrast, in the topography of the ownership effect, main differences in the N100 component lay not along the midline but bilateral temporal (Fig. 3a). Thus, the topography contributes significantly to our understanding of the differential neurobiological processes and the topographic analysis extends the literature on self-monitoring in healthy subjects.

There is, to our knowledge, no literature on electrical source imaging in the field of "sensing the self", even not in the broader scope of what we defined as agency and ownership. Therefore, we compared our ERP source estimates of the current density with the neuroimaging results gained from mainly fMRI studies. There are some imaging studies in healthy controls (Fu et al. 2006) or even schizophrenia patients, who are prone to hallucinations (Allen et al. 2007), where a paradigm comparable to our ownership effect was used: own voice and alien voice were administered via headphones after reading aloud. Activations in the bilateral insular as well as in the temporal (including the anterior parts) cortex were described ( $\mathrm{Fu}$ et al. 2006), which is comparable with our findings. We observed this increase in current density predominantly in the right hemisphere, which again is in accordance with the right sided increase when hearing the own voice in comparison to hearing an unfamiliar voice (Fu et al. 2006). 
Additionally we found decreased current density estimates in the cortical midline structures, which were also reported by $\mathrm{Fu}$ et al. (2006): they found especially the posterior located midline structures to be more activated when hearing the unfamiliar voice. Finally, we observed an increase in the current density estimates in the left inferior frontal gyrus, which might be a correlate of the reading component in our paradigm (Fiez and Petersen 1998). In an interesting study in hallucinating schizophrenia patients, exactly these cingulate regions exhibited a negative correlation between the hallucination-related coupling of the left inferior frontal gyrus and the posterior and rostral anterior cingulate cortex in dependency of the reality of auditory hallucinations (Raij et al. 2009). On the perceptual level, this is in parts comparable with our paradigm, where we observed this decrease when the unfamiliar voices where replayed.

In the second contrast agency+ versus agency- the regions with increased current density estimates in the presence of agency indicated an involvement of structures being affected by having caused an action, here, in reading the read word (irrespective of hearing the own or an unfamiliar voice). We identified decreased activation of the anterior cingulate and medial prefrontal cortex, when the subject was the cause of the percept. This is nicely in accordance with the literature on error monitoring (Van Veen and Carter 2002) and other paradigms challenging the self-monitoring system-independent of the modalities e.g. also while tickling oneself, which is anchored in the motor-sensory system (Blakemore et al. 2000). In addition, in healthy controls, functional connectivity between the medial prefrontal cortex and the temporal gyrus was found to increase when other-compared to self-generated words were processed. Schizophrenia patients showed an inverse pattern (Wang et al. 2011). The loss of the feeling of agency (in a motor-sensory study) was related to bilateral but right accented temporal activations (Nahab et al. 2011), which confirms our right temporal finding of decreased current density estimates with the loss of being the actor.

As a limitation of the present study and as an outlook on possible follow-ups, one may in addition assess the behavioural effects of agency and ownership. Furthermore, it may be interesting to look at EEG gamma synchronization (Kottlow et al. 2012), since this has been related to impaired corollary discharge mechanisms in patients with schizophrenia (Koenig et al. 2012). A further limitation might be the masking of potential interesting effects by the motor movement artefacts, which have been removed by subtracting the "Read-Aloud-Only" condition. However, since the ERP that might have been confounded by speech related artefacts displayed the typical topography of auditory components, we assume that such residual artefacts have not played a relevant role in our analyses.
Self-monitoring is a complex process with at least two main components: agency and ownership. These components are neurobiologically distinct processes. Our results support previous findings and extend our knowledge of the mechanisms to establish the 'sense of self' for spoken words. The classical central N100 suppression is determined by the agency effect, while ownership yields bilateral temporal differences. This finding may be relevant to the study of symptom clusters in schizophrenia, which are partly characterized by a deficient'sense of self' (e.g. verbal hallucinations, ego-disturbances such as thought insertion and withdrawal, or feelings of being made). We suggest that loss of agency alone may lead to symptoms of ego-disturbances. Additionally, if ownership is affected, hallucination-type symptoms may result.

Acknowledgments We are grateful to Miranka Wirth for providing the word stimuli. Further, we thank Nicolas Moor for testing out different delays.

\section{References}

Allen P, Amaro E, Fu CH, Williams SC, Brammer MJ, Johns LC, McGuire PK (2007) Neural correlates of the misattribution of speech in schizophrenia. Br J Psychiatry 190:162-169

Baess P, Horvath J, Jacobsen T, Schroger E (2011) Selective suppression of self-initiated sounds in an auditory stream: an ERP study. Psychophysiology 48(9):1276-1283

Behroozmand R, Larson CR (2011) Error-dependent modulation of speech induced auditory suppression for pitch-shifted voice feedback. BMC Neurosci. doi:10.1186/1471-2202-12-54

Blakemore SJ, Wolpert D, Frith C (2000) Why can't you tickle yourself? NeuroReport 11:R11-R16

Blakemore SJ, Wolpert DM, Frith CD (2002) Abnormalities in the awareness of action. Trends Cogn Sci 6:237-242

Crapse TB, Sommer MA (2008) Corollary discharge across the animal kingdom. Nat Rev Neurosci 9:587-600

Curio G, Neuloh G, Numminen J, Jousmaki V, Hari R (2000) Speaking modifies voice-evoked activity in the human auditory cortex. Hum Brain Mapp 9:183-191

Dierks T, Linden DE, Jandl M, Formisano E, Goebel R, Lanfermann H, Singer W (1999) Activation of Heschl's gyrus during auditory hallucinations. Neuron 22:615-621

Eichele T, Nordby H, Rimol LM, Hugdahl K (2005) Asymmetry of evoked potential latency to speech sounds predicts the ear advantage in dichotic listening. Brain Res Cogn Brain Res 24:405-412

Feinberg I (1978) Efference copy and corollary discharge: implications for thinking and its disorders. Schizophr Bull 4:636-640

Fiez JA, Petersen SE (1998) Neuroimaging studies of word reading. PNAS USA 95:914-921

Ford JM, Mathalon DH, Heinks T, Kalba S, Faustman WO, Roth WT (2001a) Neurophysiological evidence of corollary discharge dysfunction in schizophrenia. Am J Psychiatry 158:2069-2071

Ford JM, Mathalon DH, Kalba S, Whitfield S, Faustman WO, Roth WT (2001b) Cortical responsiveness during talking and listening in schizophrenia: an event-related brain potential study. Biol Psychiatry 50:540-549 
Ford JM, Gray M, Faustman WO, Roach BJ, Mathalon DH (2007) Dissecting corollary discharge dysfunction in schizophrenia. Psychophysiology 44:522-529

Ford JM, Mathalon DH (2012) Anticipating the future: automatic prediction failures in schizophrenia. Int $\mathbf{J}$ Psychophysiol 83:232-239

Fu CH, Vythelingum GN, Brammer MJ, Williams SC, Amaro E Jr, Andrew CM, Yaguez L, van Haren NE, Matsumoto K, McGuire PK (2006) An fMRI study of verbal self-monitoring: neural correlates of auditory verbal feedback. Cereb Cortex 16:969-977

Gould L, Cummine J, Borowsky R (2012) The cognitive chronometric architecture of reading aloud: semantic and lexical effects on naming onset and duration. Front Hum Neurosci 6:287

Heinks-Maldonado TH, Mathalon DH, Gray M, Ford JM (2005) Finetuning of auditory cortex during speech production. Psychophysiology 42:180-190

Heinks-Maldonado TH, Mathalon DH, Houde JF, Gray M, Faustman WO, Ford JM (2007) Relationship of imprecise corollary discharge in schizophrenia to auditory hallucinations. Arch Gen Psychiatry 64:286-296

Helmholtz Hv (1924) Helmholtz's treatise on physiological optics. Optical Society of America, New York

Holst Ev (1954) Relations between the central nervous system and the peripheral organs. Br J Anim Behav 2:89-94

Houde JF, Jordan MI (1998) Sensorimotor adaptation in speech production. Science 279:1213-1216

Houde JF, Nagarajan SS, Sekihara K, Merzenich MM (2002) Modulation of the auditory cortex during speech: an MEG study. J Cogn Neurosci 14:1125-1138

Hubl D, Koenig T, Strik WK, Garcia LM, Dierks T (2007) Competition for neuronal resources: how hallucinations make themselves heard. Br J Psychiatry 190:57-62

Jackson JH (1958) Selected writings of John Hughlings. Basic Books, New York

Johns LC, McGuire PK (1999) Verbal self-monitoring and auditory hallucinations in schizophrenia. Lancet 353(9151):469-470

Jung TP, Makeig S, Westerfield M, Townsend J, Courchesne E, Sejnowski TJ (2000) Removal of eye activity artifacts from visual event-related potentials in normal and clinical subjects. Clin Neurophysiol 111:1745-1758

Kalckert A, Ehrsson HH (2012) Moving a rubber hand that feels like your own: a dissociation of ownership and agency. Front Hum Neurosci 6:40

Koenig T, Kottlow M, Stein M, Melie-Garcia L (2011) Ragu: a free tool for the analysis of EEG and MEG event-related scalp field data using global randomization statistics. Comput Intell Neurosci 2011:938925

Koenig T, van Swam C, Dierks T, Hubl D (2012) Is gamma band EEG synchronization reduced during auditory driving in schizophrenia patients with auditory verbal hallucinations? Schizophr Res 141:266-270

Kottlow M, Jann K, Dierks T, Koenig T (2012) Increased phase synchronization during continuous face integration measured simultaneously with EEG and fMRI. Clin Neurophysiol 123:1536-1548
Leube DT, Knoblich G, Erb M, Grodd W, Bartels M, Kircher TT (2003) The neural correlates of perceiving one's own movements. Neuroimage 20:2084-2090

Macphee GJ, Crowther JA, McAlpine CH (1988) A simple screening test for hearing impairment in elderly patients. Age Ageing 17:347-351

Maidhof C, Vavatzanidis N, Prinz W, Rieger M, Koelsch S (2010) Processing expectancy violations during music performance and perception: an ERP study. J Cogn Neurosci 22:2401-2413

McCarthy-Jones S, Trauer T, Mackinnon A, Sims E, Thomas N, Copolov DL (2012) A new phenomenological survey of auditory hallucinations: evidence for subtypes and implications for theory and practice. Schizophr Bull. doi:10.1093/schbul/sbs 156

Michel CM, Koenig T, Brandeis D (2009) Electrical neuroimaging in the time domain. In: Michel CM, Koenig T, Brandeis D, Gianotti LRR, Wackermann J (eds) Electrical neuroimaging. Cambridge University Press, New York, pp 111-143

Nahab FB, Kundu P, Gallea C, Kakareka J, Pursley R, Pohida T, Miletta N, Friedman J, Hallett M (2011) The neural processes underlying self-agency. Cereb Cortex 21:48-55

Pantev C, Hoke M, Lehnertz K, Lutkenhoner B, Anogianakis G, Wittkowski W (1988) Tonotopic organization of the human auditory cortex revealed by transient auditory evoked magnetic fields. Electroencephalogr Clin Neurophysiol 69:160-170

Pascual-Marqui RD (2002) Standardized low-resolution brain electromagnetic tomography (sLORETA): technical details. Methods Find Exp Clin Pharmacol 24(Suppl D):5-12

Raij TT, Valkonen-Korhonen M, Holi M, Therman S, Lehtonen J, Hari R (2009) Reality of auditory verbal hallucinations. Brain 132:2994-3001

Sperry RW (1950) Neural basis of the spontaneous optokinetic response produced by visual inversion. J Comp Physiol Psychol 43(6):482-489

Strik WK, Fallgatter AJ, Brandeis D, Pascual-Marqui RD (1998) Three-dimensional tomography of event-related potentials during response inhibition: evidence for phasic frontal lobe activation. Electroencephalogr Clin Neurophysiol 108:406-413

Synofzik M, Vosgerau G, Voss M (2013) The experience of agency: an interplay between prediction and postdiction. Front Psychol 4:127

van Veen V, Carter CS (2002) The anterior cingulate as a conflict monitor: fMRI and ERP studies. Physiol Behav 77:477-482

Wang L, Metzak PD, Woodward TS (2011) Aberrant connectivity during self-other source monitoring in schizophrenia. Schizophr Res 125:136-142

Wirth M, Horn H, Koenig T, Razafimandimby A, Stein M, Mueller T, Federspiel A, Meier B, Dierks T, Strik W (2008) The early context effect reflects activity in the temporo-prefrontal semantic system: evidence from electrical neuroimaging of abstract and concrete word reading. Neuroimage 42:423-436

Wirth M, Jann K, Dierks T, Federspiel A, Wiest R, Horn H (2011) Semantic memory involvement in the default mode network: a functional neuroimaging study using independent component analysis. Neuroimage 54:3057-3066

Wurtz RH (2008) Neuronal mechanisms of visual stability. Vision Res 48:2070-2089 